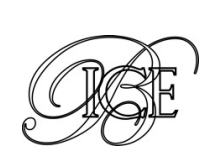

\title{
Kelly Hattel*
}

\section{LA INCLUSIÓN FINANCIERA EN FILIPINAS}

Filipinas es un referente a nivel mundial por su marco normativo para facilitar el acceso a los servicios financieros y promover la inclusión financiera. Sin embargo, tan sólo el 31,3 por 100 de los adultos posee una cuenta en una institución financiera formal y sólo cuatro de cada diez adultos filipinos destinan dinero al ahorro. La tecnología, acompañada de la educación financiera y de una eficaz protección de los consumidores, tiene potencial para romper las barreras a la difusión y dar mayores oportunidades y seguridad a los pobres de Filipinas.

Palabras clave: Filipinas, inclusión financiera, microfinanzas, microseguros.

Clasificación JEL: G21, G22, G28, O16.

\section{Contexto del país}

Situado en el sudeste de Asia, Filipinas es un archipiélago compuesto por 7.107 islas con una superficie total de aproximadamente 300.000 kilómetros. Dividido en tres regiones principales, Luzón, Visayas y Mindanao, la población del país en 2015 se estimaba en 101,6 millones de filipinos, con prácticamente la misma distribución entre hombres y mujeres. El crecimiento demográfico en las Filipinas es alto, del 1,9 por 100 anual. A pesar de una alta tasa de crecimiento económico, la pobreza persiste debido a la tensión de una población en rápido crecimiento y a los limitados recursos financieros. En la primera mitad de 2014, la incidencia de la pobreza ascendía al 25,8 por 100, ligeramente superior al 24,6 por 100 en el mismo periodo de 2013, debido fundamentalmente a un aumento de los precios de los alimentos y a una serie de graves desastres naturales, principalmente tifones ${ }^{1}$. En

\footnotetext{
* Financial Sector Specialist, South East Asia Department, Banco Asiático de Desarrollo.

Las opiniones de la autora no reflejan necesariamente las de la institución.

Versión de marzo de 2016.

1 Asian Development Bank (2015).
}

2013 el Índice de Desarrollo Humano (IDH, un indicador del progreso humano y de la compleja relación entre renta y bienestar) era un 16,5 por 100 superior al de 1980. Sin embargo, el país ocupaba el puesto 117 de los 187 países. Gracias a la positiva evolución del consumo privado positivo, la sólida perspectiva de la inversión y las exportaciones y la recuperación del gasto público, se prevé que el PIB de Filipinas aumente en un 6,3 por 100 en 2016, la cifra más alta de la región y muy por encima de la media prevista (5,3 por 100). Tras las elecciones generales que se celebrarán en mayo de 2016, el Gobierno tendrá que centrarse en el fortalecimiento de la posición fiscal y mejorar el clima de inversión².

\section{Inclusión financiera}

Los datos actuales muestran que hay unos dos mil millones de adultos en edad de trabajar en todo el mundo que no tienen acceso a servicios financieros formales. Estos individuos se apoyan en mecanismos informales de crédito, ahorro y seguros. Aunque estos mecanismos pueden ayudar $\square$

\footnotetext{
2 Asian Development Bank (2015)
} 
a las personas a salir adelante a diario, implican un riesgo y a menudo son muy caros.

Como señala el Consultative Group to Assist the Poor (CGAP), «cada vez hay mayor evidencia empírica de que los servicios financieros adecuados pueden ayudar a mejorar el bienestar de los hogares y estimular la actividad de las pequeñas empresas. También existe evidencia macroeconómica que demuestra que las economías con un mayor grado de intermediación financiera tienden a crecer más rápido y a reducir la desigualdad de la renta» ${ }^{3}$.

En la década de 1970 se introdujo un concepto más formal de los microcréditos en varias regiones del mundo, incluidos el sur de Asia y América del Sur. El experimento más notable con el microcrédito se produjo en Bangladesh en 1974, cuando Muhammad Yunus comenzó a prestar a los pobres. El programa luego se convirtió en el Banco Grameen, que en 2011 atendía a 8,3 millones de clientes $^{4}$. Estos primeros experimentos mostraron que pequeñas cantidades de capital a corto plazo en forma de créditos (microcréditos) podrían ayudar a los pobres a salir de la pobreza. El concepto de microfinanzas -prestación de servicios financieros a personas de bajos ingresos- evolucionó con el tiempo para referirse de forma general a un amplio conjunto de servicios financieros adaptados a las necesidades de las personas pobres. El término «inclusión financiera» reconoce la importancia no sólo de las microfinanzas sino también de la educación financiera, el fortalecimiento de la capacitación financiera de los consumidores y las políticas de protección al consumidor adaptadas a las necesidades de los pobres.

\section{La inclusión financiera en Filipinas}

Las microfinanzas en Filipinas se iniciaron en la década de 1950 con bancos rurales y cooperativas que proporcionaban pequeños préstamos para los trabajadores agrícolas y pescadores. Sin embargo,

\footnotetext{
3 http://www.cgap.org/topics/financial-inclusion.

4 www.grameen.com (datos de 2014). El 97 por 100 de esos clientes son mujeres.
}

los proveedores de servicios no fueron capaces de sostener los programas de préstamo debido a las bajas tasas de reembolso y a problemas de diseño de los programas. Durante la década de 1970 y hasta mediados de la década de 1980 el Gobierno comenzó a ofrecer créditos muy subvencionados a la población rural pobre a través de bancos rurales impulsados por el Gobierno, bancos de desarrollo y otras instituciones financieras gubernamentales. Hacia finales de los ochenta comenzaron a desarrollarse las organizaciones no gubernamentales (ONG) orientadas a las microfinanzas, que utilizaban metodologías alternativas para proporcionar préstamos no garantizados e instrumentos de ahorro para los pobres. En 1989, 27 instituciones participaron en uno de los primeros programas de réplica del Banco Grameen que se hicieron en el mundo, y que puso en marcha el Agricultural Credit Policy Council, organismo afiliado del Departamento de Agricultura (MCPI Annual Report). Con el tiempo, muchos bancos rurales en Filipinas evolucionaron para proporcionar servicios financieros a las poblaciones marginadas. Los bancos rurales de Filipinas comenzaron también a ofrecer servicios de microfinanzas, en parte a través de la cooperación con el programa de Acceso de Microempresas a Servicios Bancarios (Microenterprise Access to Banking Services o MABS) iniciado en 1998 y ejecutado por la Asociación de Banqueros Rurales de las Filipinas (Rural Bankers Association of the Philippines o RBAP). En 2013 había 9.884 oficinas bancarias y 14.528 cajeros automáticos en el país, así como 182 bancos con operaciones de microfinanzas que proporcionaban una amplia gama de servicios financieros (ahorro, crédito, pagos, seguros, remesas e inversiones). En marzo de 2016 las instituciones de microfinanzas englobadas en el MIX Market ${ }^{5}$ reflejaban una cartera conjunta de créditos por valor de 1.400 millones de dólares repartidos entre 5,1 millones de prestatarios, y de 923,2 millones en depósitos aportados por 7,2 millones de depositantes ${ }^{6}$.

\footnotetext{
${ }^{5}$ El MIX Market (www.mixmarket.org) es un centro de datos donde las instituciones de microfinanzas (IMF) y sus organismos de apoyo comparten datos institucionales para ampliar la transparencia y el conocimiento del mercado.

${ }^{6}$ http://www.mixmarket.org/mfi/country/Philippines\#ixzz41dAnouMO
} 
Al mismo tiempo, las políticas gubernamentales en las dos últimas décadas han llevado a cabo reformas para agilizar las microfinanzas en Filipinas. La primera Estrategia Nacional para las Microfinanzas la desarrolló en 1997 el Consejo Nacional de Crédito (National Credit Council o NCC), un organismo interinstitucional bajo el Departamento de Hacienda creado en 1993. Esta primera estrategia pretendía crear un mercado de microfinanzas privado viable y sostenible que se extendería a servicios financieros para hogares de bajos ingresos y microempresas. La Ley General Bancaria, aprobada en 2000, reconoció las microfinanzas como una actividad bancaria legítima y, lo que es más importante, facultó al banco central (Bangko Sentral ng Pilipinas o BSP) para desarrollar un marco propicio para la regulación del sector.

Hoy en día, el sector de las microfinanzas incluye una variedad de proveedores regulados y no regulados, tales como bancos, cooperativas de crédito, las ONG microfinancieras, casas de empeño y agentes de dinero electrónico que ofrecen una amplia gama de servicios financieros al público. El BSP promueve un entorno propicio para la inclusión financiera ${ }^{7}$ a través de la adopción de diversas reglamentaciones y la emisión de circulares que fomentan la entrada de nuevos proveedores de servicios financieros y la creación de productos adecuados para los pobres, garantizando al mismo tiempo la prestación segura de dichos servicios. Reconocido entre los reguladores como un líder en la creación de un entorno propicio para la inclusión financiera, en 2014 el Banco Central de Filipinas (BSP) fue galardonado con el Premio Maya Declaration de la Alianza para la Inclusión Financiera (Alliance for Finance Inclusion) por sus compromisos tangibles de inclusión financiera. Asimismo, en 2015 Filipinas ocupaba el primer puesto de Asia y el tercero a nivel mundial (detrás de Perú y Colombia) en términos de entorno propicio para la inclusión financiera, de

\footnotetext{
7 La inclusión financiera o un sistema financiero inclusivo se define como un estado en el que existe un acceso efectivo a una amplia gama de productos y servicios financieros por parte de todos.
}

acuerdo con la encuesta mundial de entornos de inclusión financiera del Economist Intelligence Unit (EIU 2015).

\section{Otros logros importantes}

Además de los productos de crédito y ahorro, Filipinas ha estado en la vanguardia del desarrollo de otras áreas importantes de la inclusión financiera como microseguros, educación financiera y protección del consumidor y dinero electrónico.

\subsection{Microseguros}

Desde que se puso en marcha en 2010 el Marco Estratégico y Regulatorio Nacional para los Microseguros, el microseguro en Filipinas ha experimentado un crecimiento exponencial, habiendo actualmente 28 millones de personas cubiertas por 89 productos de microseguros aprobados por la Comisión de Seguros. Se han desarrollado extensos canales de distribución para bancos, cooperativas, las ONG microfinancieras e individuos que actúan como agentes acreditados o corredores. Los seguros (salud, vida, propiedad) proporcionan protección contra el riesgo financiero para las personas. En 2013, la cobertura de seguro de vida en Filipinas alcanzaba tan sólo al 32,5 por 100 de la población. El gasto medio por persona en seguros pasó de 18 dólares en 2009 a cerca de 44 dólares en 2013. Existen necesidades crecientes de seguros contra el riesgo de desastres y agrícolas en el segmento de bajos ingresos, dada la propensión a los desastres de Filipinas, que se ve afectada anualmente por tifones e inundaciones. El devastador tifón Hayan en 2013, que afectó sobre todo a la ciudad de Tacloban, subrayó la necesidad de un seguro y de un sistema justo y eficiente de pago de las reclamaciones. A 11 de febrero de 2014 se habían producido 11.633 reclamaciones de microseguros por parte de asegurados en zonas afectadas, lo que equivale a 72,3 millones de pesos filipinos pagados a los beneficiarios $^{8}$. Si bien la penetración de seguros del $D$

\footnotetext{
8 CARD MRI.
} 
país pasó del 1 por 100 en 2009 al 1,8 por 100 en 2013, el volumen de primas en porcentaje del PIB de Filipinas sigue siendo relativamente bajo ${ }^{9}$.

\subsection{Educación financiera y protección del consumidor}

EI BSP lleva mucho tiempo comprometido en la promoción de la educación financiera en Filipinas y la implementación de medidas para la protección del consumidor. En octubre de 2006 se aprobó la orden de creación del Grupo de Asuntos Financieros del Consumidor (Financial Consumer Affairs Group o FCAG) dentro del Sector de Supervisión y Examen (Supervision and Examination Sector 0 SES). El grupo se creó para apoyar al SES a garantizar la protección de depositantes e inversores $y$ favorecer un funcionamiento fluido $y$ ordenado de todo el sistema financiero. Desde entonces, el BSP ha llevado a cabo programas de educación financiera en todo el país, colaborando con las entidades locales (Local Government Units o LGUs) y las oficinas provinciales y organismos implicados. Los programas de educación financiera del BSP tienen como objetivo fomentar una toma de decisiones económicas y financieras bien informada por parte de los clientes, mejorar la comprensión de los servicios financieros y de sus derechos y obligaciones, y ayudar a los proveedores financieros a cumplir con las regulaciones relacionadas con la protección de los consumidores. La oficina también cuenta con un mecanismo de asistencia al consumidor a modo de sistema de reclamaciones para canalizar las quejas y compensaciones de los consumidores.

\subsection{Dinero electrónico (e-Money)}

En 2009, el BSP emitió la Circular BSP 649 que establece las directrices sobre transacciones con dinero electrónico. En 2010 había 21 emisores de

\footnotetext{
9 Insurance Commission (2014).
}

dinero electrónico registrados (Electronic Money Issueres o EMIs). En 2013, el número de EMIs se había incrementado un 52 por 100, hasta los 32. En ese mismo período el número de cuentas registradas de dinero electrónico pasó de 19,9 millones a 26,7 millones, lo que supone un aumento del 34 por 100. Dichas transacciones se mantienen en forma de 8 millones de billeteras móviles y 18,7 millones de tarjetas de efectivo. El número de transacciones de dinero electrónico también ha crecido significativamente en los últimos años: de los 138 millones de transacciones en dinero electrónico en 2010 se pasó a 217 millones en 2013 (57 por 100 de aumento). En términos cuantitativos, el valor total de las transacciones de dinero electrónico aumentó un 58 por 100 entre 2010 y 2013, pasando de 220,5 millones a 348 millones de pesos. El número de agentes activos de dinero electrónico creció un 20 por 100, alcanzando los 10.620 agentes en 2013 frente a los 8.819 de $2010^{10}$. Estos pagos móviles fueron utilizados para una amplia gama de actividades, como los pagos interpersonales (P2P), las transferencias de dinero ${ }^{11}$, el comercio electrónico móvil (m-commerce), transacciones móviles en puntos de venta (POS), nómina electrónica y pago de facturas.

\section{Resultados: un gran progreso, pero todavía una baja inclusión financiera}

A pesar del impresionante crecimiento de las microfinanzas y de tener un marco de desarrollo para la inclusión financiera de reconocimiento mundial, el nivel de inclusión financiera de Filipinas sigue siendo bajo. Hay una restricción regulatoria para las entidades no bancarias de microfinanzas a la hora de prestar determinados servicios generales como depósitos, créditos, pagos, remesas, inversiones y $\triangleright$

\footnotetext{
10 Bangko Sentral ng Filipinas (2014).

11 En Filipinas son muy relevantes las transferencias interpersonales de remesas. Así, en 2013 el 38 por 100 de los adultos filipinos recibía remesas de un familiar en el extranjero. Con más de 10 millones de filipinos en el extranjero en 2013 (Commission on Filipinos Overseas, 2015), las remesas alcanzaron un nivel equivalente al 8,5 por 100 del Producto Interior Bruto en 2014. Alrededor del 39 por 100 de los hogares que reciben remesas asignan una parte al ahorro.
} 


\begin{tabular}{|c|c|c|}
\hline \multicolumn{3}{|c|}{$\begin{array}{c}\text { TABLA } 1 \\
\text { INCLUSIÓN FINANCIERA EN EL SUDESTE ASIÁTICO } \\
\text { (Porcentaje en 2014) }\end{array}$} \\
\hline País & $\begin{array}{l}\text { Porcentaje de adultos con cuenta en una } \\
\text { institución financiera formal } \\
\text { (todos) }\end{array}$ & $\begin{array}{l}\text { Porcentaje de adultos con cuenta en una } \\
\text { institución financiera formal } \\
\text { (40 por } 100 \text { de menor renta) }\end{array}$ \\
\hline Singapur & 96,4 & 96,2 \\
\hline Malasia & 80,7 & 75,6 \\
\hline 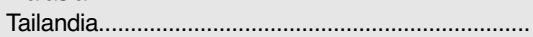 & 78,1 & 72,0 \\
\hline 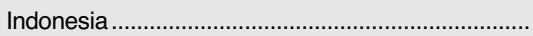 & 36,1 & 22,2 \\
\hline 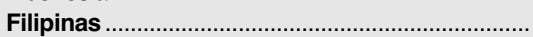 & 31,3 & 17,8 \\
\hline Vietnam & 31,0 & 18,9 \\
\hline
\end{tabular}

seguros, que harían frente a las necesidades multidimensionales de servicios financieros eficaces por parte de individuos, familias y empresas en todo el país. El porcentaje de adultos que mantiene una cuenta en una institución financiera formal es del 31,3 por 100. Al mismo tiempo y de forma desproporcionada, la población con menores ingresos está económicamente excluida, ya que en los quintiles de ingresos que suponen el 40 por 100 de los adultos con menores ingresos, tan sólo el 17,8 por 100 tiene una cuenta en una institución financiera formal. Por otro lado, sólo cuatro de cada diez adultos filipinos apartan dinero para ahorrar, y el 68 por 100 de los que ahorran mantienen sus ahorros en casa. Un 33 por 100 tiene ahorros guardados en bancos, un 7,5 por 100 en cooperativas y un 2,6 por 100 en sistemas de ahorro de grupo ${ }^{12}$. La disparidad en el acceso a servicios financieros formales por nivel de renta es mayor en Filipinas que en otros países de la región, como muestra la Tabla 1.

A finales de diciembre de 2014, un 36 por 100 de los municipios no tenían oficina bancaria ${ }^{13}$. Por tanto, el acceso financiero presenta una enorme disparidad regional, con una fuerte concentración de oficinas bancarias en las regiones altamente pobladas. Las áreas no atendidas por los bancos están parcialmente cubiertas por las instituciones financieras no bancarias, como las ONG microfinancieras, cooperativas, casas de empeño, agentes de remesas, proveedores de servicios de pago electrónico y agentes de banca móvil. Sin embargo,

\footnotetext{
12 Bangko Sentral ng Pilipinas (2015).

13 lbid.
}

muchas de estas personas no tienen acceso a una amplia gama de servicios financieros.

Con más de 7.100 islas, el desafío geográfico para que los servicios financieros lleguen a la gente es tremendo. La tecnología puede ayudar a superar este reto, pero muchas personas siguen recelando de los pagos electrónicos y siguen utilizando y confiando en el efectivo.

\section{El camino hacia una mayor inclusión financiera}

Uno de los más importantes logros recientes en el sector es la adopción de una Estrategia Nacional para la Inclusión Financiera, firmada el 1 de julio de 2015 y desarrollada a través de un comité compuesto de 13 agencias gubernamentales. La estrategia establece un marco y un plan de acción para el Gobierno y el sector privado para adoptar un enfoque coordinado y sistemático para el desarrollo de un sistema financiero que sea accesible y sensible a las necesidades de toda la población. En 2015 se llevó a cabo la encuesta nacional de referencia sobre la Inclusión Financiera (Nationwide Baseline Survey on Financial Inclusion o NBSFI), primera encuesta representativa a nivel nacional dedicada a la recogida entre adultos filipinos de datos de inclusión financiera por lado de la demanda, desde la perspectiva de los usuarios reales y potenciales de los servicios financieros. Los resultados de la encuesta proporcionan una imagen más precisa del estado de la inclusión financiera en las Filipinas en términos de acceso, uso, calidad y bienestar percibido. 


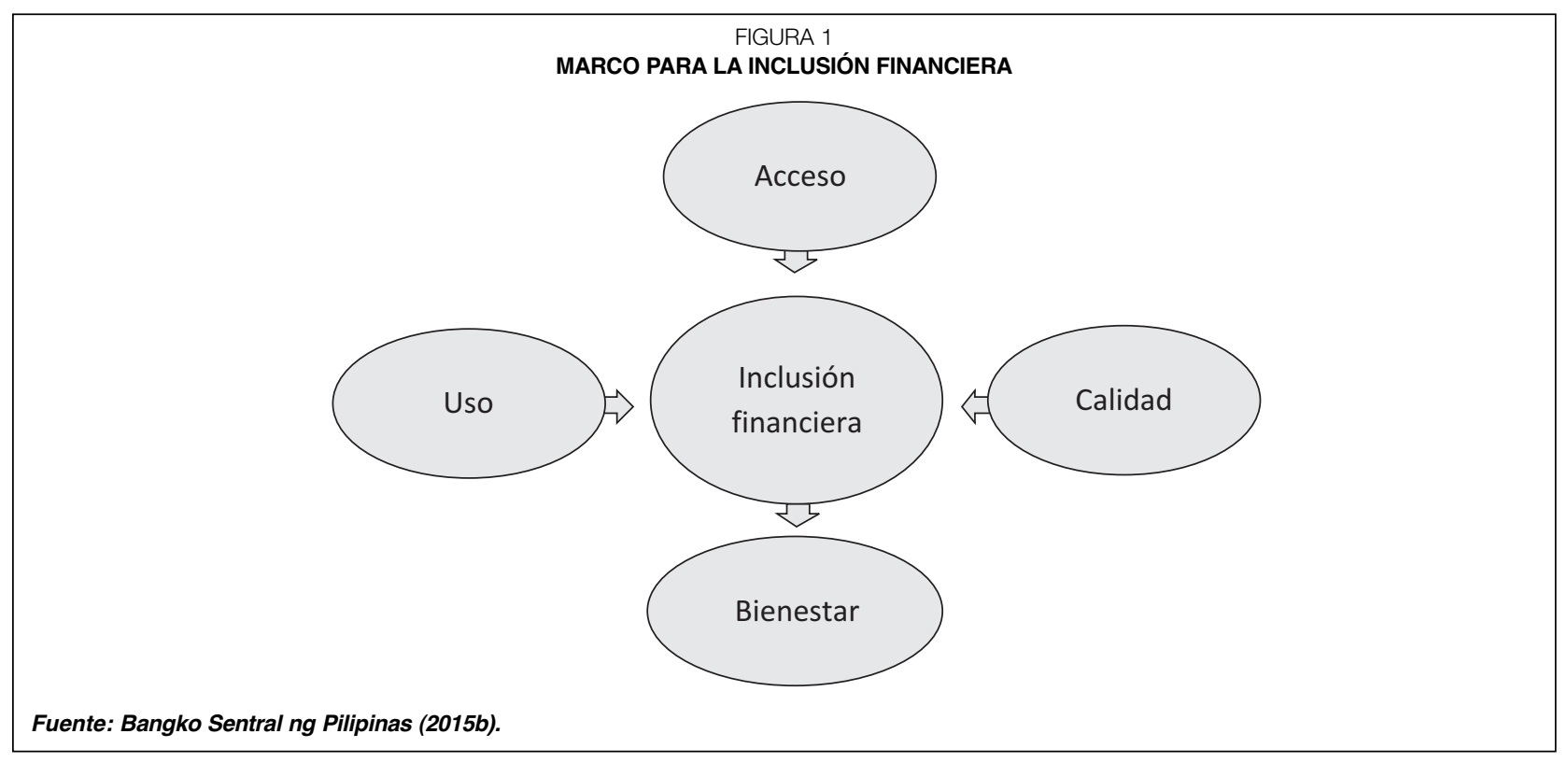

De acuerdo con el BSP, las dos frases «acceso efectivo» $y$ «amplia gama de productos y servicios financieros" son componentes clave de la definición, y cruciales para el diseño, ejecución y seguimiento de esta estrategia. «Acceso efectivo» no sólo significa que haya productos y servicios financieros que están disponibles, sino que abarca cuatro componentes más amplios que se muestran en la Figura 1. Aparte del acceso físico a los productos y servicios financieros, dichos productos y servicios deben tener un diseño adecuado, de buena calidad, y relevantes para conducir a un uso real que puede beneficiar a la persona que acceda a dicho servicio ${ }^{14}$.

La Estrategia Nacional proporciona un marco para que el Gobierno y el sector privado adopten un enfoque coordinado y sistemático hacia una visión clara del problema. El Mapa Estratégico (Strategy Map) incluye tres pilares clave con el objetivo de lograr una «inclusión financiera hacia el crecimiento de base amplia e integradora»: la regulación y supervisión de la política, la educación financiera y protección al consumidor y los programas de promoción. Los pilares se mantienen unidos por un marco efectivo de datos y de medición. La aplicación de la estrategia incluye un proceso de coordinación de las aportaciones de las partes clave

\footnotetext{
${ }^{14}$ Bangko Sentral ng Pilipinas (2015b).
}

interesadas y de los agentes del mercado, con el BSP al frente del seguimiento de la estrategia.

Las principales áreas de enfoque marco de la estrategia para aumentar la inclusión financiera en los próximos años incluyen:

- Promoción de las finanzas digitales. Los esfuerzos del BSP para facilitar medios de pago electrónicos han abierto una vía innovadora para que la población de bajos ingresos pueda realizar pagos con tarjetas prepago monederos electrónicos ${ }^{15}$. Mientras, las transacciones de venta al por menor, en su conjunto, siguen basándose principalmente en el efectivo debido a los costosos sistemas de pago nacionales, que no están unificados. Una parte importante de la estrategia es el desarrollo de un Sistema Nacional de Pagos al por Menor (National Retail Payment System) que podría revolucionar los pagos en el país. Una exitosa aplicación del NRPS incrementaría el volumen y el valor de las transacciones de pago electrónico y abriría más oportunidades para ampliar el acceso financiero mediante la vinculación de un sistema efectivo de pago electrónico con cuentas bancarias.

- Mejora de la gestión de crédito a través del sistema de información crediticia. La gestión del crédito $\triangleright$

\footnotetext{
15 Estos instrumentos de dinero electrónico han superado la falta de infraestructuras y han permitido un eficaz desembolso por parte del Gobierno de transferencias condicionadas de efectivo y una entrega rápida de dinero en efectivo a las víctimas de grandes tifones.
} 
Tendencias EN LA INCLUSión FINANCIERA EN FiLIPINAS

Las siguientes tendencias influirán en gran medida en el futuro de la inclusión financiera en Filipinas:

- La creciente demanda de financiación de cadenas de valor agrícola continuará como resultado de los desastres naturales recientes y esperados y sus efectos sobre los precios de los alimentos.

- Cada vez más bancos comerciales están reduciendo la escala de sus servicios financieros, generando una mayor competencia con las instituciones financieras de microfinanzas tradicionales, con el objetivo de aumentar su eficiencia. Sin embargo, esta competencia puede llegar a desplazar a las ONG que pueden centrarse más en el segmento más pobre de la población.

- La mayor demanda, el mayor volumen de operaciones y el aumento de las oportunidades para el uso de la tecnología en la ampliación de los servicios financieros ha incrementado la necesidad de una gama más amplia de políticas de protección de los consumidores y de actividades de educación financiera.

- A pesar de presentar una baja ratio de penetración, los productos de microseguros se han ampliado considerablemente en los últimos años y la demanda seguirá creciendo.

- El potencial para incrementar la escala en términos de acceso y uso de servicios financieros, particularmente a través de la tecnología, es grande. Sin embargo, esta innovación debe equilibrarse con una mayor educación y protección efectiva del consumidor financiero tanto para las instituciones reguladas como para las no reguladas.

- El sobreendeudamiento, especialmente en las provincias, continuará siendo una preocupación y requiere un esfuerzo concertado de toda la industria para abordar la cuestión.

requiere un sistema de información de crédito eficaz. En 2008 se aprobó la Ley del Sistema de Información de Crédito (Credit Information System Act o CISA) con el objetivo de recoger y difundir toda la información de crédito de las entidades legales para mejorar la calidad global de los préstamos y reducir el sobreendeudamiento. Para poner en práctica la CISA, en 2014 se estableció la Sociedad de Información Crediticia (Credit Information Corporation o $\mathrm{CIC}$ ), bajo la supervisión de la Comisión Nacional de Valores (Securities Exchange Commision o SEC). La CIC ha estado llevando a cabo formación para facilitar a las entidades de crédito el cumplimiento de los requisitos de la CISA para poder remitir información crediticia al CIC.

- Desarrollo de un registro de garantías muebles. Para mejorar el acceso al crédito formal por parte de las micro, pequeñas y medianas empresas (mipymes), carentes de inmuebles susceptibles de garantía, es necesario establecer un registro de garantías muebles, a través del cual los pequeños prestatarios puedan obtener el crédito necesario sobre la base de garantías alternativas como equipos, maquinaria, inventarios y cuentas por cobrar debidamente registradas. El Departamento de Hacienda ha estado estudiando la posible creación de un registro de garantías muebles, en consulta con el sector privado y agencias de cooperación.

- Fortalecimiento de las ONG microfinancieras. Aunque las ONG microfinancieras desempeñan un papel importante para el acceso de clientes tradicionalmente desatendidos o parcialmente desatendidos, no tenían un sistema de regulación prudencial. Estas ONG están obligadas tan sólo a presentar estados financieros auditados anuales y hojas de información general a la SEC, lo que pone de relieve el riesgo fiduciario dada su recogida de cuasidepósitos de clientes con un objetivo de «acumulación de capital». En respuesta a esto, en 2015 se aprobó la Ley de las ONG Microfinancieras (Microfinance NGO Act), que establece un marco normativo adecuado para que las ONG microfinancieras puedan llevar a cabo operaciones financieras sobre bases sólidas, al tiempo que se protege a los clientes financieros. La ley establece requisitos reglamentarios en las áreas de gobernabilidad, operaciones, información y protección de los consumidores que deben cumplir las ONG microfinancieras. Bajo la nueva ley, se establecerá un Consejo Regulador de las ONG Microfinancieras (Microfinance NGO Regulatory Council) bajo la autoridad de la SEC. Los próximos pasos serán los siguientes: (i) la formulación de los desarrollos reglamentarios oportunos, (ii) la difusión de la nueva regulación, (iii) el diseño de un sistema eficaz de seguimiento y procedimientos de supervisión, $y$ (iv) el fortalecimiento de la capacidad regulatoria y de cumplimiento (compliance) para lograr que los servicios prestados por las ONG de microfinanzas sean seguros, accesibles y de confianza. El nuevo reglamento facultará al BSP para inspeccionar las ONG microfinancieras y garantizar que se respetan los límites prudenciales de captación de depósitos. 
Kelly Hattel

- Desarrollo de la cadena de valor de las finanzas. El acceso a la financiación es el reto más apremiante para las mipymes para sostener y expandir sus actividades comerciales. Una financiación oportuna y suficiente en las fases críticas de la cadena de valor, incluyendo la adquisición, fabricación, procesamiento y envío, maximizaría la utilización de recursos, aumentaría las ganancias y generaría empleo. Carentes de inmuebles que sivieran de colateral y con débiles condiciones financieras, la mayoría de las mipymes no son elegibles para la financiación formal y no logran crecer. Las restrictivas normas prudenciales derivadas del último Acuerdo de Basilea impiden de forma desproporcionada el acceso a la financiación de los bancos por parte de las mipymes. Un mayor nivel de provisiones para pérdidas crediticias y de ponderación de riesgo hace que la financiación sea demasiado costosa o a menudo imposible. Sin embargo, los últimos ejemplos de préstamos con éxito a las cadenas de valor en la elaboración de alimentos pueden extenderse a otros sectores. EI BSP apoya la promoción de la financiación basada en colaterales alternativos, e intentará formular regulaciones de apoyo a la financiación de la cadena de valor en la agricultura dentro de la nueva estrategia de inclusión financiera.

\section{Conclusión}

El alcance de la inclusión financiera en Filipinas se ha expandido enormemente en los últimos años. Aunque Filipinas ha liderado la promoción y la creación de un entorno propicio para la inclusión financiera, lo cierto es que sólo el 26 por 100 de los filipinos adultos tienen cuentas de ahorro y sólo el 10,5 por 100 tiene acceso al crédito formal. Este bajo nivel de penetración sigue siendo un reto. El Gobierno actual ha supuesto un gran apoyo a las microfinanzas, aunque las próximas elecciones podrían dar lugar a un nuevo Gobierno con diferentes prioridades políticas. La adopción en 2015 de la Estrategia Nacional para la Inclusión Financiera ${ }^{16}$, con el compromiso de aceptación por parte de una serie de agencias gubernamentales y de grupos de interés, es un hito importante para continuar los progresos realizados para ampliar la inclusión financiera. La tecnología desempeñará un papel clave en esta expansión, pero los canales nuevos y alternativos deberán ser cuidadosamente equilibrados con la protección del consumidor y la educación eficaz de los consumidores. Con la base sólida que se ha construido en las últimas décadas, un marco regulador fuerte y una estrategia clara, cada vez más y más filipinos tendrán un mayor acceso y capacidad de utilización de unos servicios financieros que les ayuden a mejorar sus vidas, protegerse contra crisis inesperadas y planificar su futuro.

${ }^{16}$ Los estudios han demostrado que las naciones con estrategias sólidas para la inclusión financiera han tenido más éxito en la consecución de sus objetivos de inclusión financiera.

\section{Bibliografía}

[1] ASIAN DEVELOPMENT BANK (2015). Asian Development Outlook. Manila.

[2] BANGKO SENTRAL NG PILIPINAS (2014). Report on the State of Financial Inclusion in the Philippines. Manila.

[3] BANGKO SENTRAL NG PILIPINAS (2015). National Baseline Survey on Financial Inclusion. Manila.

[4] BANGKO SENTRAL NG PILIPINAS (2015b). National Strategy for Financial Inclusion. Manila.

[5] COMMISSION ON FILIPINOS OVERSEA (2015). CFO Annual Report 2014-2015.

[6] ECONOMIST INTELLIGENCE UNIT (2015). Global Microscope 2015: The Enabling Environment for Economic Inclusion. Disponible en: www.centerforfinancialinclusion.org

[7] INSURANCE COMMISSION (2014). 2013 Annual Report. Manila. 